\title{
Editorial
}

\section{Citas y autocitas}

드튜 ET

TE배튜
El nivel de una revista científica puede ser valorada en función de indicadores de calidad, estos constituyen una métrica para cuantificar la aceptación de la revista dentro de la comunidad académica. Uno de estos mide los resultados obtenidos, entre los que se destaca el número de acceso o número de artículos descargados, así como el número de citas bibliográficas recibidas por los trabajos publicados en la revista, entre los que se destaca el factor de impacto y el índice de inmediatez [1].

En Colombia existen en la actualidad más de 40 Journal nacionales reconocidos y clasificados por Colciencias que publican periódicamente, trabajos resultados de investigación, relacionados con la ingeniería. Muchos de estos son de acceso libre y pueden ser descargados en cualquier momento, sin embargo, ¿cuáles son los resultados de estas publicaciones en el ámbito local? ¿Los artículos publicados en revistas nacionales de ingeniería están siendo referenciados en otros trabajos posteriores, también publicados en revistas de ingeniería nacionales? ¿Existe alguna dinámica acerca del número de citas recibidas por año? ¿Cómo el comportamiento del nivel de auto y no auto cita? 
En el presente editorial se realiza un breve estudio exploratorio para cuantificar la dinámica de citas recibidas por nuestra revista Scientia et Technica. Para ello se revisaron la sección de Bibliografía de los artículos publicados en 45 revistas de Ingenieria nacionales en el periodo comprendido entre el 2007 - 2015. Las referencias fueron clasificadas en Auto y No Auto citas [2] y el proceso de recopilación de citas se llevó acabo, por medio de planeación de búsqueda y palabras claves sugerido en la metodología de vigilancia tecnológica [3]. A continuación (figura 1) se enseña un gráfico representando la dinámica de citas totales a nivel nacional (líneas continuas) y citas totales logradas en la propia revista para cuatro casos de estudio (círculos). Es de notar que la dinámica de citas totales de Scientia Et Technica (ver descripción de la figura) presenta un comportamiento a la baja, debido a que la citas en su propia revista ha disminuido considerablemente. En caso contrario se tiene el comportamiento de dos revistas colombianas de gran impacto, las cuales presentan una tendencia positiva en la dinámica total de citas recibidas, lo anterior se debe al incremento considerable en las citas recibidas en su propio Journal.

Figura 1: Dinámica total de citas por año, línea continua indica número total de citas identificadas y los puntos indican el número total de citas identificadas en la propia revista. Color azul lo llamaremos Revista 1, línea roja Scientia et Technica, Revista 2 linea verde y Revista 3 linea negra. Es de notar que las Revista 1, 2 y 3, son revistas reales, a las cuales no se les da el nombre real debido a problemas de marca registrada.

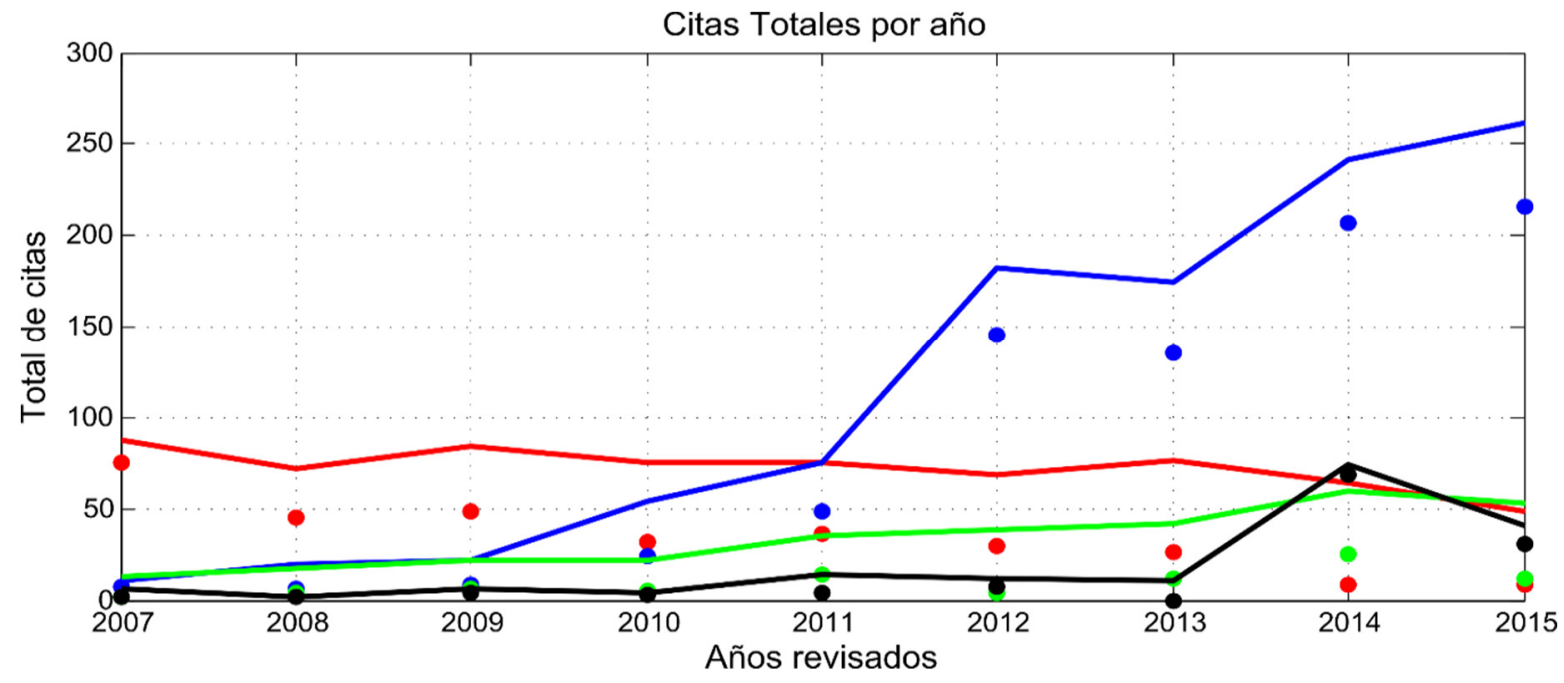

Por otro lado, a continuación (figura 2) se enseña el gráfico correspondiente al comportamiento de las citas auto. Nótese como en la revista Scietia Et Technica la tendencia en el número de auto citas es a la baja, lo anterior se 
debe a gran medida a la disminución de estas en la propia revista. En los casos de la Revista 3 la Revista 1, la tendencia es positiva.

Figura 2: Dinámica de auto citas por año. Línea continua indica número total de auto citas identificadas y los puntos indican el número de auto citas identificadas en la propia revista. Nuevamente identificaremos las revistas así: Color azul lo llamaremos Revista 1, linea roja Scientia et Technica, Revista 2 linea verde y Revista 3 línea negra.

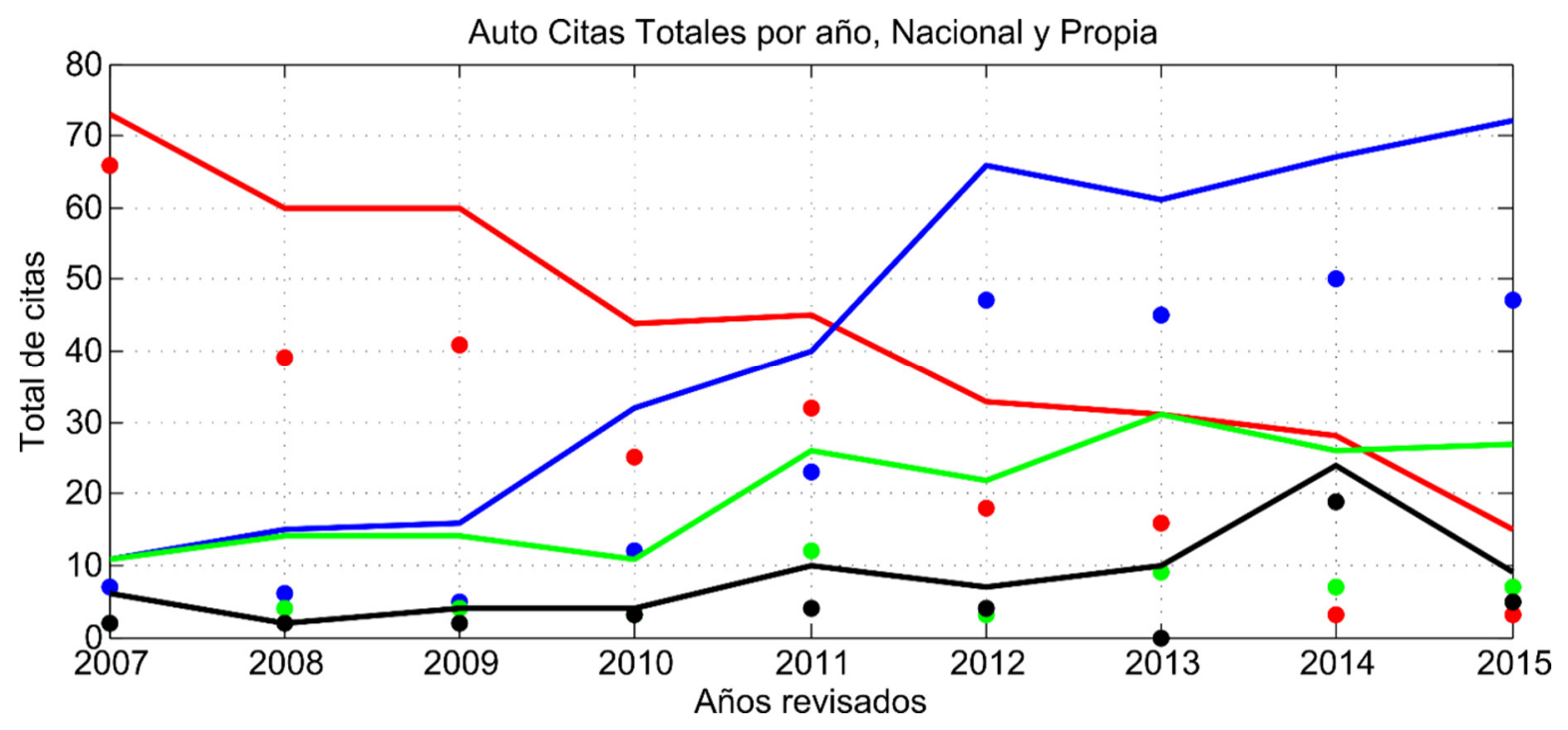

Por último, se presenta la dinámica de las citas no auto, tanto para el total general como el discriminativo (el número de no auto citas logrado en otras revistas sin tener en cuenta la propia revista). Se observa que Scientia eT Technica y en la Revista 2, el número de no auto citas es en su mayoría es alcanzado en otras revistas. Contrario a los demás casos de estudio donde el número de no auto citas predomina en la propia revista.

Figura 3: Dinámica de No auto citas por año, la línea continua indica número total de No auto citas identificadas y los puntos indican el número de No auto citas identificadas en otras revistas (exceptuando la propia revista). color azul Revista 1, linea roja Scientia et Technica, linea verde Revista 2, linea negra Revista 3. 


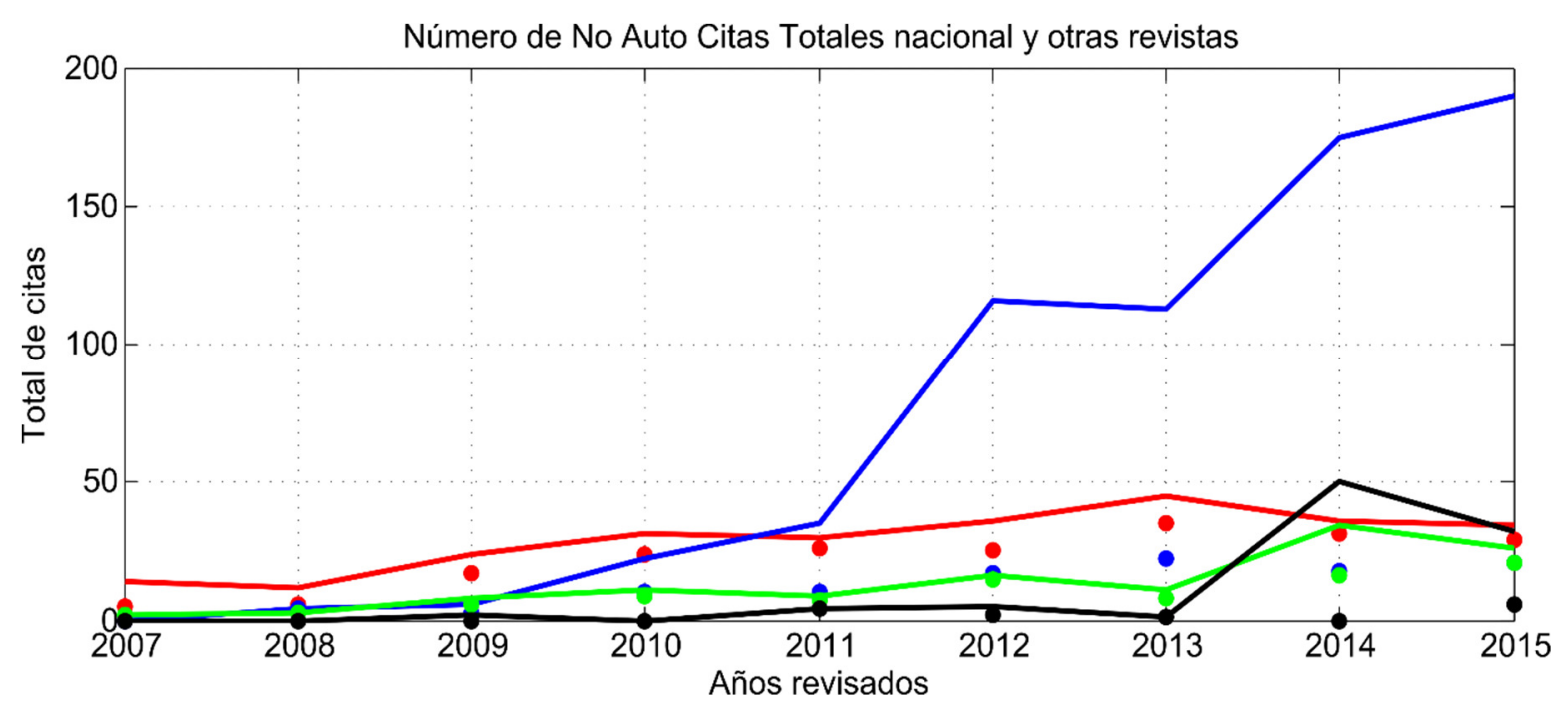

Este pequeño estudio no pretende ser exhaustivo, sin embargo, muestra algunos patrones interesantes para determinar el impacto de una revista nacional a nivel local. En el caso de la revista Scientia Et Technica muestra que el número total de citas recibidas en 45 editoriales nacionales de ingeniería, presenta una tendencia a la baja, esto se debe en gran medida a la disminución de las citas auto recibidas en su propia revista.

Por otro lado, el gráfico de la figura 3 sugiere que los artículos publicados por el comité editorial de revista Scientia Et Technica están siendo consumidos por autores que publican en otras revistas de ingeniería nacional. Es muy importante como estrategia fortalecer la revista a nivel interno en la Universidad Tecnológica de Pereira, a partir de trabajos inéditos de alto nivel con el propósito de que revista sea un referente para los investigadores de la región que publican en Scientia Et Technica.

\section{Referencias}

[1] MIYAHIRA ARAKAKI, Juan M. Scientific journals: quality criteria. Rev Med Hered [online]. 2008, vol.19, n.1.

[2] INFORMACION TECNOLOGICA. Las Autocitas en Artículos de Revistas de Corriente Principal. Inf. tecnol. [online]. 2008, vol.19, n.5.

[3] Escorsa, P., Maspons, R., \& Llibre, J. (2001). De la vigilancia tecnológica a la inteligencia competitiva. Prentice hall. 


\section{Autores}

\section{Carlos Alberto Henao Baena}

Gestor Línea de Electrónica y Telecomunicaciones, Tecnoparque Nodo Pereira

Andrés Felipe Calvo Salcedo

Director Programa de Ingeniería Electrónica, Universidad Tecnológica de Pereira

Hugo Armando Gallego Becerra

Editor Scientia Et technica, Universidad Tecnológica de Pereira 